INPLASY

PROTOCOL

To cite: Liu et al. Traditional Chinese medicine therapies for cervical spondylotic radiculopathy: A protocol for Bayesian network metaanalysis. Inplasy protocol 2021100114. doi:

10.37766/inplasy2021.10.0114

Received: 29 October 2021

Published: 29 October 2021

Corresponding author:

Yuxin Liu

853605556@qq.com

Author Affiliation:

Beijing University of Chinese Medicine

Support: National Key R\&D Program.

Review Stage at time of this submission: Piloting of the study selection process.

\section{Traditional Chinese medicine therapies for cervical spondylotic radiculopathy: A protocol for Bayesian network meta-analysis}

\author{
Liu, YX1; Zhai, SQ2; Liu, XC3; Liu, JT44.
}

Review question / Objective: P: Patients diagnosed with cervical spondylotic radiculopathy (CSR). I: The treatmen group will be treated with traditional Chinese medicine therapies (with/without additional treatment) including acupuncture of several types (acupuncture, acupoint sticking, acupoint injection, electroacupuncture and so on), massage, cervical traction, fire needle, traditional Chinese medicine hot compress, or their combination. No restrictions are imposed on length of the treatment period, times of treatment, and frequency of treatment. C: The traditional Chinese medicine therapies (with/without additional treatment) including acupuncture of several types (acupuncture, acupoint sticking, acupoint injection, electroacupuncture and so on), massage, cervical traction, fire needle, traditional Chinese medicine hot compress, or their combination will be included in this review. S: Only randomized controlled trials (RCTs) will be included.

INPLASY registration number: This protocol was registered with the International Platform of Registered Systematic Review and Meta-Analysis Protocols (INPLASY) on 29 October 2021 and was last updated on 29 October 2021 (registration number INPLASY2021100114).

Conflicts of interest:

None declared.

\section{INTRODUCTION}

Review question / Objective: P: Patients diagnosed with cervical spondylotic radiculopathy (CSR). I: The treatmen group will be treated with traditional Chinese medicine therapies (with/without additional treatment) including acupuncture of several types (acupuncture, acupoint sticking, acupoint injection, electroacupuncture and so on), massage, cervical traction, fire needle, traditional Chinese medicine hot compress, or their combination. No restrictions are imposed on length of the treatment period, times of treatment, and frequency of treatment. C: The traditional Chinese medicine therapies (with/without additional treatment) including 
acupuncture of several types (acupuncture, acupoint sticking, acupoint injection, electroacupuncture and so on), massage, cervical traction, fire needle, traditional Chinese medicine hot compress, or their combination will be included in this review. S: Only randomized controlled trials (RCTs) will be included.

Condition being studied: Cervical spondylotic radiculopathy (CSR) is the most common type of cervical spondylosis, accounting for about $60 \%$ of the incidence of cervical spondylosis. The existing randomized controlled trials (RCTs) suggest that traditional Chinese medicine therapies (including cervical traction, fire needle, traditional Chinese medicine hot compress, etc) may have potentials in the treatment of CSR, but to date, controversy still exists among practitioners regarding the Chinese medicine approach to be used for the treatment of CSR. Therefore, this study will systematically evaluate the efficacy and safety of traditional Chinese medicine therapies in the treatment of CSR.

\section{METHODS}

Search strategy: A retrieval will be performed in 8 online databases (the Cochrane Library, Web of Science, PubMed, EMBASE Database, China Biological Medicine Database, Chinese Scientific Journals Database, Wan Fang databases, and China National Knowledge Infrastructure) and 2 trial registration platforms (ClinicalTrials.gov and Chinese Clinic Trials.gov) from their inception throughout December 2021. Only randomized controlled trials of testing traditional Chinese medicine therapies for CSR will be enrolled, regardless of language.

Participant or population: Patients diagnosed with cervical spondylotic radiculopathy (CSR).

Intervention: The treatmen group will be treated with traditional Chinese medicine therapies (with/without additional treatment) including acupuncture of several types (acupuncture, acupoint sticking, acupoint injection, electroacupuncture and so on), massage, cervical traction, fire needle, traditional Chinese medicine hot compress, or their combination. No restrictions are imposed on length of the treatment period, times of treatment, and frequency of treatment.

Comparator: The traditional Chinese medicine therapies (with/without additional treatment) including acupuncture of several types (acupuncture, acupoint sticking, acupoint injection, electroacupuncture and so on), massage, cervical traction, fire needle, traditional Chinese medicine hot compress, or their combination will be included in this review.

Study designs to be included: Randomized controlled trials (RCTs) will be included.

Eligibility criteria: Articles will be included if they are independent original RCTs of good quality.

Information sources: 8 online databases (the Cochrane Library, Web of Science, PubMed, EMBASE Database, China Biological Medicine Database, Chinese Scientific Journals Database, Wan Fang databases, and China National Knowledge Infrastructure) and 2 trial registration platforms (ClinicalTrials.gov and Chinese Clinic Trials.gov).

Main outcome(s): Visual analogue scale (VAS).

Additional outcome(s): Cervical range of motion, assessment of muscle strength, neck disability index (NDI) scores, the MOS item short from health survey, activities of daily living, total efficiency, and safety assessments.

Data management: Two researchers will extract the data independently. A data extraction table, which mainly includes first author's name, nationality, publication year, participants' characteristics (sample size, gender, mean age, number of groups, disease duration, and so on), interventions, comparators, outcomes, and 
methodological design will be established in Microsoft Excel 2019 in light of recommendations in the Cochrane Handbook. Data for study information, which include type of study, country where the study was conducted, sample size of the study, length of follow-up period, and interventions will be collected using standard data extraction methods.

Quality assessment / Risk of bias analysis: Two well-trained researchers will independently assess the bias risk of all the included RCTs by the Cochrane Collaboration tool, which consisted of the following aspects: assignment concealment, random sequence generation, blinding of outcome assessors, blinding of participants and personnel, selective reporting, the integrity of outcome data, and other sources of bias. Each field has been classified as high risk, low risk, or unclear risk. Any disagreements will be resolved by discussion with a third senior assessor.

Strategy of data synthesis: In each of the studies, the relative risk (RR) will be calculated for dichotomous outcomes (e.g., total complications), and treatment effects for continuous outcomes (e.g., VAS and NDI score) including mean differences (MDs) for studies with comparable outcome measures used a $95 \%$ confidence interval(Cl). STATA Version 12.0 (Stata Corp, College Station, TX), an open and free statistics software, will be utilized in data synthesis. We choose the random effects model to perform pairwise meta-analysis for all the available outcomes in this study. Standardized MD for continuous outcomes and RRs for dichotomous outcomes will be used as summary statistics, with corresponding $95 \% \mathrm{Cl}$. The Bayesian NMA was performed on the Markov chain Monte Carlo method in WinBUGS statistical software Version 1.4.3. To rank the interventions for CSR, we will implement the surface under the cumulative ranking curve (SUCRA). The percentage is the most common form of expression for the SUCRA that ranged from $0 \%$ (or 0 ) to $100 \%$ (or 1 ). It is clearly understood that highest (namely, $100 \%$ or 1 ) or lowest SUCRA (namely, $0 \%$ or 0) represents the best and the worst treatment, respectively. Inconsistencies of this NMA will be assessed by the nodesplitting analysis. Significance levels $<0.05$ will be considered evidence of inconsistency. Results of different random effects and fixed effects models were compared for the sensitivity analysis.

Subgroup analysis: First of all, we will assess if the participants' characteristics, interventions, as well as outcomes are adequately similar in all included trials. If not, the heterogeneity of the results will be assessed with I-square (12) by STATA 12.0. The substantial heterogeneity will be considered only if the $I 2$ is above $50 \%$, and then a random-effects model will be chosen, otherwise a fixed-effects model will be used. In general, differences across dissimilar kinds of evidence may be a major source of inconsistency. Thus, the inconsistency between indirect and direct evidence will be assessed by the loop inconsistency test and node-splitting method. Additionally, Z-value as well as corresponding $P$ value will be calculated, and $P$ values less than 0.05 demonstrate $a$ significant difference. In case significant heterogeneity is identified, subgroup analysis will be performed according to the possible sources of heterogeneity, such as quality of studies.

Sensitivity analysis: Considering that varying level of the methodological quality of trails may tend to affect our findings, sensitivity analysis will be performed to evaluate the robustness of the results by excluding high-risk studies.

Language: No limits of language will be imposed on the search.

Country(ies) involved: China.

Keywords: network meta-analysis; cervical spondylotic radiculopathy; traditional Chinese medicine

Contributions of each author:

Author 1 - Yuxin Liu - The author developed drafted the manuscript and developed the 
methodology for this network metaanalysis.

Email: 853605556@qq.com

Author 2 - Shuangqing Zhai - The author conceived the idea for this study, he also read, provided feedback and approved the final manuscriptand and is the guarantor of this study.

Email: zsq2098@163.com

Author 3 - Xiuchao Liu - The author will screen potential studies and perform duplicate independent data extraction, risk of bias assessment, GRADE assessment and data synthesis.

Email: 287487527@qq.com

Author 4 - Jintao Liu - The author will perform duplicate independent data extraction, risk of bias assessment, GRADE assessment and data synthesis.

Email: lixdd666@gmail.com 